\title{
Flipped Classroom, dando la vuelta a una asignatura de ingeniería en la UPV
}

Ignacio Despujol, Linda Castañeda y Jaime Busquets

Ignacio Despujol Zabala, ndespujol@asic.upv.es, es Técnico de Sistemas de Nuevas Aplicaciones de Internet en el Área de Sistemas de Información y Comunicaciones (ASIC) de la Universitat Politècnica de Valencia (UPV); Linda Castañeda Quintero, lindacq@um.es, es profesora del Departamento de Didáctica y Organización Escolar en la Universidad de Murcia y Jaime Busquets Mataix, busquets@asic.upv.es, es Jefe de Servicio de Sistemas y Redes de Comunicación en el ASIC de la UPV.

\begin{abstract}
Se describe la utilización y resultados de los medios tecnológicos puestos por la UPV a disposición de los profesores para transformar al modelo "flipped" la asignatura Ingeniería Aeroportuaria, de tercer curso del grado de Ingeniería Aeroespacial, que ha contado en el curso 2015-16 con un total de 120 alumnos divididos en 2 grupos.

Se ha utilizado de forma intensiva la tecnología Polimedia para grabar la teoría de la asignatura en vídeos cortos y el formato de importación de contenidos de SakaiApereo para, utilizando una hoja Excel, automatizar su subida a la herramienta de contenidos del sistema de Campus Virtual de la UPV (PoliformaT) en un formato similar al de los MOOC. Con ello la explicación de la teoría ha sido seguida por los alumnos desde su casa y se han dedicado las clases presenciales al planteamiento de casos prácticos y su resolución en equipos de 4 o 5 estudiantes y la exposición del resultado obtenido.

También se ha utilizado el formato de importación de exámenes del LMS para, con un libro Excel, crear de forma automática baterías de problemas con valores de partida distintos por alumno para proponer las colecciones de ejercicios que los alumnos realizan a lo largo del curso y el examen final.

En la asignatura se trabajan también las competencias transversales en un trabajo final en equipo que se presenta en clase al resto de compañeros.

Finalmente, se ha pasado a los estudiantes una encuesta de valoración de la experiencia, y se presentan los resultados obtenidos.
\end{abstract}

\section{Keywords:}

Flipped Classroom, clase inversa, vídeo, Polimedia, Sakai Apereo, LMS, Higher Education, Universidad 
Flipped Classroom, dando la vuelta a una asignatura de ingeniería en la UPV

\section{Introducción}

Como puede leerse en el documento marco (Integración del sistema universitario español en el Espacio Europeo de Enseñanza Superior, 2003), el nuevo Espacio Europeo de Educación Superior, adoptado a raíz del conocido como Proceso de Bolonia, propone un modelo de enseñanza superior mucho más centrado en el estudiante y en que adquiera no sólo los conocimientos técnicos sino las competencias transversales necesarias para su incorporación a un mundo laboral cada vez más complejo, multidisciplinar y cambiante (trabajo en equipo, capacidad de adaptarse a problemas desconocidos, capacidad de aprender durante toda la vida, etc...)

La Universitat Politècnica de València, ya en su (Plan Estratégico 2007-14, 2007), decidió apostar con decisión por la incorporación de las Tecnologías de la Información a la docencia reglada, desarrollando diferentes servicios de apoyo a sus profesores para la generación de objetos digitales de aprendizaje yun proyecto específico de Docencia en Red.

Para el curso 2015/2016 la UPV ha lanzado una iniciativa ambiciosa de transformación de clases al formato de clase inversa. Con el objeto de extraer conclusiones que pudieran servir de orientación al resto de profesores participantes en la citada iniciativa, y como disponíamos de diverso material docente en vídeo, decidimos en el curso 2014-2015 generar el material adicional necesario para incorporar el formato de clase inversa en algunos de los temas de la asignatura Ingeniería Aeroportuaria (troncal de tercer curso en los estudios de Grado en Ingeniería Aeroespacial, y que ha contado este curso con dos grupos de 73 y 50 alumnos). La idea era convertirla totalmente al formato de clase inversa en el curso 2015/2016 en el marco de la iniciativa. Para ello se incluyó en la guía docente un procedimiento de evaluación que permitiera acomodar esta incorporación parcial.

Al final se ha podido impartir la práctica totalidad de la asignatura en formato inverso (sólo 4 de las sesiones siguieron el modelo tradicional), pero el tener que preparar los nuevos materiales en paralelo a la impartición ha sido un gran reto y el poco margen de tiempo disponible para la adaptación ha influido en algunos aspectos, lo que creemos que se ve reflejado en la encuesta de opinión de los alumnos y proporciona un margen de mejora claro para siguientes ediciones.

Se ha llevado a cabo un proceso de investigación-acción en el que se ha diseñado el contenido y la metodología de la asignatura y se ha ido adaptando conforme se ha ido desarrollando el curso. Se ha diseñado una encuesta de satisfacción final que nos permita extraer conclusiones y mejorar el curso para posteriores ediciones. En el momento de redactar este artículo no se ha realizado todavía el examen final, con lo que las respuestas de los alumnos no incluyen la percepción completa de los mismos sobre el curso ya que no han realizado el esfuerzo para preparar dicho examen. 


\section{Objetivos}

El objetivo principal del trabajo ha sido una prueba piloto de implantación del sistema de clase inversa en una asignatura de grado con más de 120 alumnos, creando el material, diseñando procesos y mecanismos de evaluación y estudiando la opinión de los alumnos.

Los objetivos particulares eran:

- Diseñar y optimizar un proceso de creación del material necesario para llevar a cabo una clase inversa haciendo uso de las herramientas tecnológicas de la UPV.

- Desarrollar y optimizar mecanismos automáticos de evaluación masiva.

- Crear el material.

- Estudiar los problemas que aparecieran en la implementación y desarrollo de la clase inversa y sus posibles soluciones.

- Estudiar la percepción de los alumnos del sistema de clase inversa.

- Obtener conclusiones a aplicar en los cursos siguientes.

\section{Desarrollo de la innovación}

En el proceso de adaptación de la asignatura al formato de clase inversa hemos trabajado en las siguientes áreas:

\subsection{Creación del material necesario para la clase inversa}

Para adaptar la asignatura al formato de clase inversa ha sido necesario grabar toda la teoría de la asignatura en vídeos cortos y diseñar las actividades a desarrollar en las clases y la dinámica a desarrollar en las mismas.

\subsubsection{Grabación de los vídeos y subida a PoliformaT}

Se decidió utilizar el sistema Polimedia (Turró, Cañero, Busquets, 2010), propio de la UPV, como formato para los vídeos de la asignatura, e intentar hacerlos lo más cortos que fuera posible.

Las transparencias utilizadas el curso anterior servían a su vez como apuntes, con lo que algunas de ellas no eran óptimas para la grabación de vídeos por contener excesivo texto; se tuvieron por tanto que rehacer pasando el texto a la zona de notas y buscando imágenes representativas (Creative Commons o libres de derechos siempre que fue posible), para luego publicar en el apartado de recursos un documento con sólo las transparencias y otro con las transparencias y las notas.

Para grabar el total de 13,5 horas de vídeo se emplearon 32,6 horas de estudio, lo que da unos 25 minutos de vídeos cortos por cada hora de estudio de grabación. 
La asignatura es una asignatura descriptiva que, aunque sólo tiene sólo 4,5 créditos, consta de un temario muy extenso ( 23 temas) al condensar todo lo relativo a los aeropuertos que ven los alumnos en la carrera si no eligen la especialidad. En total se han grabado 213 vídeos (208 polimedias y 5 screencasts) de los temas 2 al 23 (el tema 1 se imparte en modo tradicional el primer día de clase), con 808 minutos de vídeo (unas 13,5 horas) y una media de 3 minutos 47 segundos por vídeo (con un vídeo de 16 minutos, 2 vídeos de entre 16 y 13 minutos, 4 vídeos de entre 13 y 10 minutos y 13 vídeos de entre 10 y 7 minutos).

Se pretendía preparar una pregunta tipo test para después de cada vídeo que sirviera como evaluación formativa ( vídeos, pero sólo se pudieron hacer 110 (temas 2 al 12 y 22 y 23).

Dada la gran cantidad de vídeos y preguntas generadas, se diseñó un formato parecido al de un curso MOOC para incorporar el material al apartado de contenidos de PoliformaT y un proceso para subir el contenido de la manera más automatizada que fuese posible.

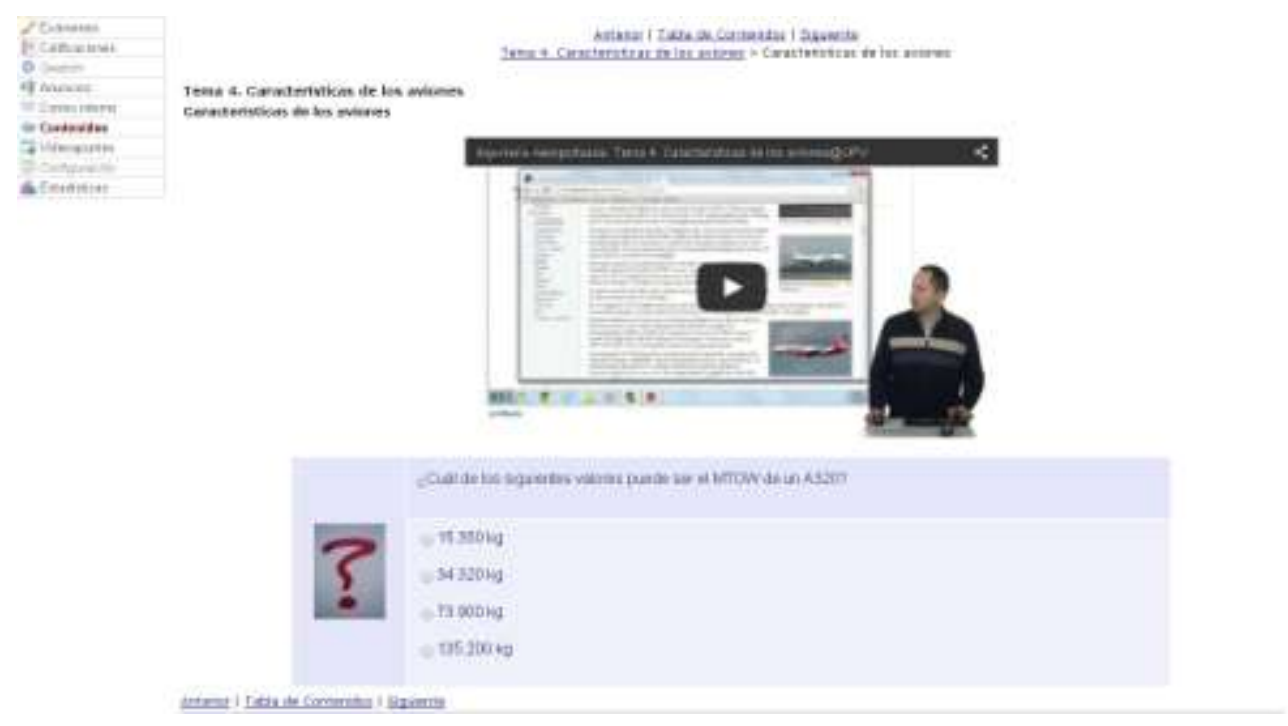

Fig. 1 Herramienta de contenidos en PoliformaT

Los vídeos fueron subidos a Youtube y añadidos allí a una lista de reproducción con un doble objeto: que estuvieran agrupados en el canal y poder automatizar la captura de los códigos de los vídeos en Youtube para subir sus referencias a PoliformaT.

Para estructurar el contenido y subirlo posteriormente a PoliformaT se utilizó una hoja Excel en la que se incluyen los códigos de los vídeos, sus títulos y los campos necesarios para incorporar la pregunta de evaluación formativa (opcional).

2015, Universitat Politècnica de València

Congreso IN-RED (2015) 


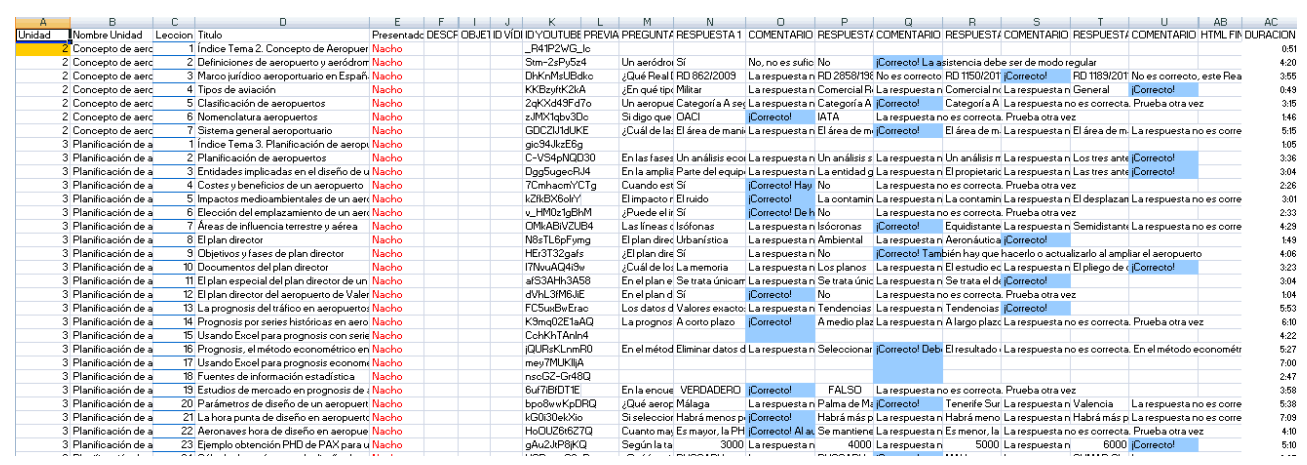

Fig. 1 Hoja de cálculo con el contenido del MOOC

El proceso de incorporación de contenido a PoliformaT tiene 2 fases:

La primera es añadir el nuevo contenido a la hoja a partir de la información de los vídeos subidos a Youtube. Para ello se copia la información de la lista de reproducción y se pega en una hoja de Excel en blanco. Con una macro de Excel se crea una función que extrae el hipervículo de una celda y con esa función y las funciones de extraer y formatear texto se prepara una hoja en la que está el título del vídeo, el de la unidad, el código del vídeo y su duración, datos que luego se incorporan a la hoja. A continuación se añaden los campos de la pregunta de refuerzo en la misma hoja.

Para este proceso (repasar los vídeos, subirlos a Youtube, añadir títulos y demás metadatos, extraer los datos de Youtube, preparar la hoja de cálculo, añadir las preguntas de refuerzo, etc.) se han empleado otras 33 horas (2,5 horas por hora de vídeo).

La segunda parte es convertir el contenido de la hoja en un formato que pueda ser importado en PoliformaT. Para ello en el libro de Excel se han incluido unas hojas intermedias que generan el código HTML a cargar en el editor de PoliformaT para conseguir tener el vídeo incrustado y la pregunta. Este código puede pegarse a mano en el editor de la plataforma, algo que resulta tedioso y que consume mucho tiempo si se tienen que incorporar muchos vídeos. Por ello creamos una macro que empaqueta en formato IMS las unidades que seleccione el profesor, para luego importarlas con la herramienta disponible en PoliformaT. La hoja de cálculo genera en el mismo directorio un paquete con las unidades seleccionadas que puede incorporarse directamente con la herramienta de importación de contenidos de PoliformaT.

3.1.2. Diseño del contenido de las clases presenciales y su dinámica

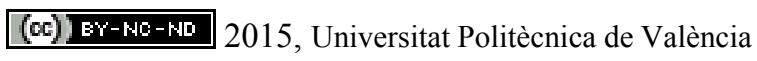

Congreso In-Red (2015) 
Además de preparar el contenido teórico para que los alumnos trabajen en casa, el formato de clase inversa requiere la preparación de la dinámica de las clases y de los problemas y casos a desarrollar en ellas para reforzar y complementar los conceptos.

Decidimos implementar una dinámica de clase totalmente inversa en la que proponer casos prácticos o problemas y que los alumnos constituyeran grupos de 4 o 5 personas para resolverlos (no era obligatorio que fueran los mismos cada vez). Aquellos que acabaran primero la actividad la exponían al resto de sus compañeros, lo que contaba para la nota final. Además, al acabar la actividad debían subir el trabajo realizado cada uno a su espacio compartido de la plataforma mientras estaban en clase o justo después, de forma que sirviera como control de asistencia a la clase. Si el trabajo realizado era en papel se les pedía que hicieran una foto con el móvil y la subieran al espacio compartido.

La idea inicial era utilizar los trabajos de clase únicamente para controlar la asistencia (haciendo que los subieran a Poliformat durante la clase o al acabar ésta), pero dada la calidad de alguno de los trabajos expuestos, consideramos que debía contemplarse de alguna manera la calidad de lo subido en el apartado de la nota reservada al portfolio, lo que, dado el gran número de alumnos, plantea problemas de corrección.

Para intentar que los alumnos vinieran a clase habiendo visto los vídeos, se les dijo que mientras estaban trabajando el profesor seleccionaría a un número de ellos (entre 5 y 10) y les asignaría uno de los conceptos correspondientes a la teoría de la sesión, dejándoles 5 minutos para prepararlo y teniéndolo que exponer luego a la clase, lo que contaría para nota. En la práctica se realizaron unas cuantas sesiones de control (aproximadamente en un $25 \%$ de las clases).

El trabajo propuesto en alguna de las sesiones consistía en confeccionar una hoja de cálculo que resolviera el problema. Como alguno de las funciones a incorporar es compleja, en lugar de optar por una resolución guiada del problema, se grabó un vídeo con la confección de la hoja de cálculo con un conjunto de datos distinto al que se utilizó luego en clase.

Se mantuvo una actividad que ya realizaron los alumnos de cursos anteriores diseñado para que trabajen varias competencias transversales: los alumnos se organizan en grupos de 5 alumnos (4 en algún caso) a los que se les asigna la confección de un informe ejecutivo sobre el Plan Director de un aeropuerto español. Disponen de 15 caras de folio para resumir lo que consideren más importante del mismo y 4 caras adicionales para comparar la situación actual con lo que se decía en el plan director. Luego disponen de 15 minutos en clase para exponer ante sus compañeros el trabajo realizado.

También se proyectaron algunos vídeos de la operación de los aviones en el aeropuerto que ilustran las razones de la normativa que estudian en clase.

Los trabajos propuestos para trabajar en clase fueron: 
Tabla 1. Trabajos en la clase

\begin{abstract}
Construir gráficas mensuales de los movimientos de pasajeros y de aeronaves en los aeropuertos españoles con los datos de AENA y la dirección general de aviación civil

Calcular la previsión de tráfico de un aeropuerto a 10 años con el método de proyección de series históricas

Calcular la previsión de tráfico de un aeropuerto a 10 años con el método de proyección econométrico seleccionando las variables que influyen en el tráfico
\end{abstract}

Calcular diversos valores de tráfico de un aeropuerto (pasajeros anuales, pasajeros por meses, mes más cargado, semana tipo, día más cargado, pasajeros hora punta, pasajeros hora de diseño) a partir de una tabla con los datos de pasajeros por hora

Calcular los pasajeros hora de diseño de un aeropuerto a partir de los datos de pasajeros anuales con las fórmulas publicadas por la FAA

Ejercicio de cálculo de distancias declaradas de una pista

Obtener distancias de despegue de un avión para un aeropuerto concreto con las fórmulas genéricas y las gráficas que hay en el manual de aeropuertos del avión

Obtener los rangos posibles de velocidad de decisión (V1) de un avión en un aeropuerto determinado

Decidir la orientación óptima de una pista a partir de un fichero de frecuencias e intensidades de viento usando Excel

Calcular la temperatura de referencia de aeródromo de un aeropuerto en Denia a partir de los datos de temperatura disponibles en internet

Ejercicio de determinación de la distancia mínima entre cambios de pendiente en la pista

Ejercicio de visibilidad en pista, comprobación de cumplimiento de normativa

Dimensionamiento completo del área de maniobras de un aeropuerto, incluidas las calles de rodaje

Ejercicios simples de determinación de dimensiones de superficies de protección de obstaculos y servidumbres radioeléctricas

Determinación de la superficie compuesta de protección de obstáculos y servidumbres radioeléctricas de un aeropuerto pequeño. Dibujo de la superficie en 2D o 3D

Diseño de señales pintadas en pista y sistema de iluminación de pista, calles de rodaje y aproximación ILS

Ejercicio de dimensionamiento del Servicio de Emergencias y Extinción de incendios del aeropuerto de Palma

Resumen del real decreto que regula el handling en España y búsqueda de compañías de handling por aeropuerto

Comparación del tráfico de aeronaves de 4 aeropuertos cargueros y de pasajeros de la red de AENA

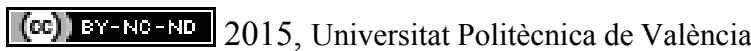

Congreso In-Red (2015) 
Flipped Classroom, dando la vuelta a una asignatura de ingeniería en la UPV

Descripción de las ULD (Unit Load Devices) usada en carga aérea

Confección del esquema básico de la red de media tensión propuesta para el aeropuerto de Albacete

Confección del esquema de la central eléctrica y los centros de transformación de la red eléctrica del aeropuerto de Albacete

Determinar la capacidad del Aeropuerto de Palma con las normas gráficas de la FAA

Ejercicio de determinación de distancias andando en terminal aeroportuario con distintas configuraciones en la ubicación de los aviones con conexiones

Ejercicio de comparación de costes de construir una pasarela de embarque frente a usar autobuses

Visita al aeropuerto de Valencia a ver in situ lo tratado en el curso

\subsection{Evaluación automática}

Uno de los grandes problemas de aplicar metodologías activas a las asignaturas con grupos numerosos de alumnos es la gran cantidad de ejercicios que deben ser corregidos por el profesor durante el curso. Esto no es específico de la metodología de clase inversa, sino que se da en cualquier sistema en el que se pretenda controlar el trabajo realizado por los alumnos a lo largo del curso. Por ello, ya en cursos anteriores, desarrollamos un sistema que nos permite generar colecciones de problemas personalizadas utilizando la herramienta de exámenes de PoliformaT. Utilizamos el mismo sistema para generar el examen final de la asignatura con preguntas con datos distintos para cada estudiante.

El sistema está basado en utilizar una hoja de cálculo de Excel que, haciendo uso de las funciones aleatorias y las fórmulas internas, permite generar tantas versiones distintas de un mismo problema como queramos. No es necesario que los problemas tengan una solución numérica que se obtenga con una fórmula, pues con el uso de tablas y las funciones de índice como BUSCARV se pueden crear problemas basados en buscar un dato en una columna de una tabla y recuperar la solución de otra columna.

También hemos usado otras hojas de cálculo adicionales para generar conjuntos de datos distintos, guardarlos por separado en ficheros y resolver un problema concreto para esos datos, creando listas de pares de ficheros de datos y soluciones que luego podemos tratar con las funciones de búsqueda comentadas. Hemos subido los ficheros con la herramienta Webdav a PoliformaT y generado problemas con un fichero de datos distinto por alumno.

La hoja de cálculo permite también incluir varias respuestas por problema y tolerancias en las preguntas de respuesta numérica. En cada ejercicio el profesor decide el número de problemas distintos a generar. 
Al ejecutar una macro, la hoja de cálculo genera una batería de problemas por ejercicio con el número de problemas seleccionado y guarda cada batería en un fichero IMS QTI que puede ser importado directamente desde la herramienta de exámenes de PoliformaT:

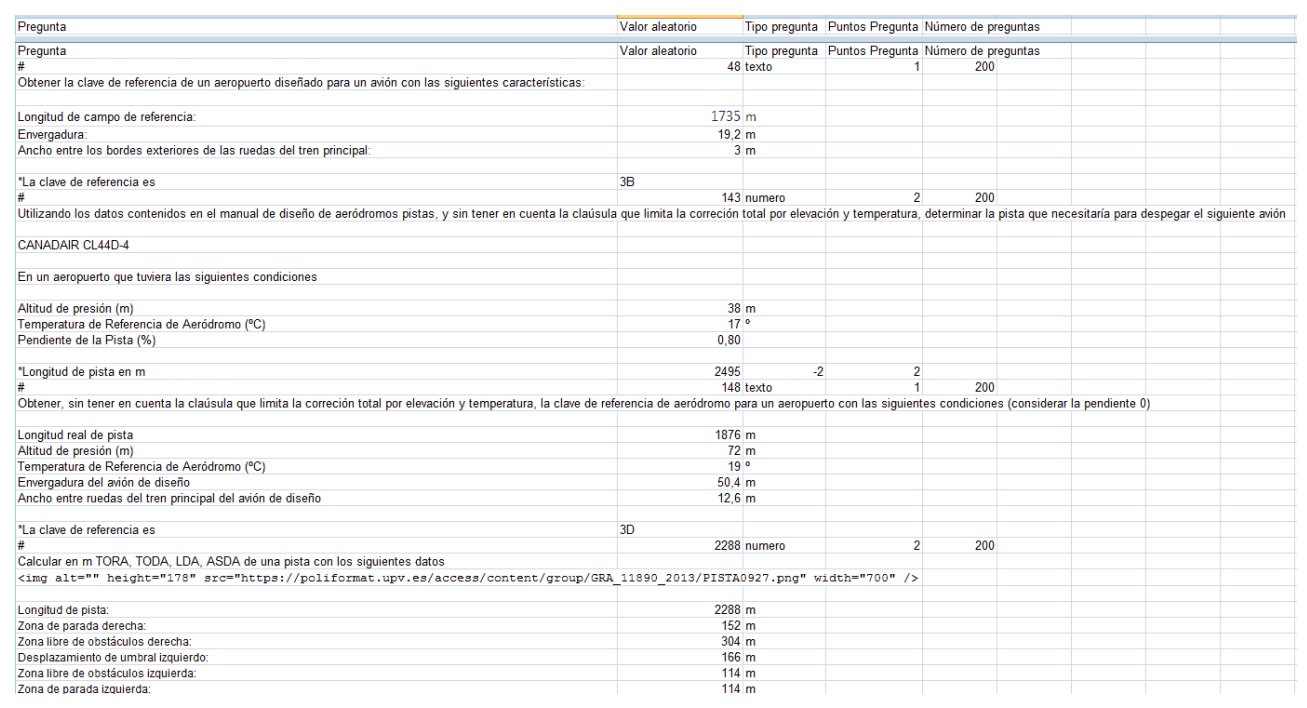

Fig. 2 Herramienta de generación de exámenes

Con las baterías generadas hemos creado un examen por cada colección de problemas a entregar, en el que los alumnos tienen 3 intentos por problema.

\subsection{Problemas de implementación}

Tras analizar los problemas encontrados creemos que pueden agruparse en 3 tipos:

\subsubsection{Problemas inherentes al formato de clase inversa}

Consideramos que los principales problemas, detectados en la encuesta de satisfacción, tienen que ver con las costumbres y percepciones de los alumnos del sistema español de educación superior, ambas fruto del funcionamiento del sistema actual hasta ahora.

El primero de ellos, reflejado en las respuestas de texto abierto de los alumnos, es la falta de capacidad de autorregulación en muchos de los estudiantes que, si no tienen una obligación de visualizar los vídeos que se refleje en su nota, acaban no viéndolos antes de ir a clase, con la consiguiente pérdida de tiempo (no pueden realizar los casos propuestos) y la acumulación de tareas para el final de curso. Para atacar este problema se incluyó la pregunta de algunos de los conceptos de los vídeos comentada anteriormente. Tras observar el comportamiento de los alumnos en clase y con la idea de dejar más tiempo para la solución de los casos prácticos y problemas, sólo se realizaron unas cuantas sesiones de control, lo que, a tenor de las respuestas de los alumnos en la encuesta, fue un error.

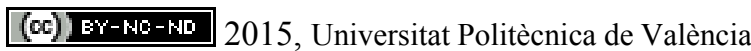

Congreso In-Red (2015) 
Por otro lado, como se refleja en (Conejero, Vendrell, Terrasa y Sanchís, 2006), creemos que los alumnos universitarios actuales están acostumbrados a dedicar a las asignaturas menos tiempo del que ellos mismos creen que es necesario (que, por otra parte, es similar al estimado en el sistema de créditos ECTS del Espacio Europeo de Educación Superior).

En la adaptación de esta asignatura al formato de clase inversa se trabajó con el valor de 30 horas de dedicación del alumno por crédito ECTS (Guía docente de la UPV: Criterios para su elaboración, UPV 2006), lo que, para una asignatura de 4,5 créditos nos da un valor de 135 horas. Teniendo en cuenta que hay:

16 semanas lectivas con 3 horas de clase a la semana (48 horas)

13,5 horas de vídeo, que pueden requerir hasta 3 horas de estudio cada una (40,5 horas)

1 trabajo de resumen de plan director (15 horas de trabajo individual y 10 en grupo)

12 problemas a resolver (6 horas)

Obtenemos un total de 119,5 horas, lo que deja 15,5 horas para preparar los exámenes finales (escrito y oral) en los que se preguntan los conceptos más básicos de la asignatura, enumerados por el profesor en clase. Estos cálculos no fueron comentados al principio de la asignatura y, como veremos en los resultados de la encuesta, la percepción de los alumnos ha sido que han trabajado demasiado para los 4,5 créditos de la asignatura.

\subsubsection{Problemas derivados de aplicar este formato a una clase con más de 120 alumnos}

Hemos detectado 2 problemas a la hora de utilizar este formato en clases masivas, el primero es que se genera una ingente cantidad de material a revisar por el profesor y el segundo es que es difícil realizar un seguimiento personalizado en una clase de 75 alumnos, con lo que el profesor puede acabar trabajando principalmente con los alumnos más implicados, lo que supone una pérdida de motivación de los demás.

\subsubsection{Problemas generados por las prisas en la implementación}

La generación del material a la vez que se desarrollaba el curso y la condición de profesor asociado del docente han hecho que, en algunos casos, los vídeos hayan estado a disposición de los alumnos sólo un par de días antes de las clases y que,el profesor no haya podido dedicar el tiempo suficiente a analizar día a día lo que estaba sucediendo en clase e implementar mejoras en tiempo real con las conclusiones obtenidas en clases anteriores.

\subsection{Percepción de los alumnos}

Han contestado a la encuesta 104 de los 123 alumnos. Sólo 92 (91 en alguna pregunta) han emitido opinión.

Tabla 2. Preferencias sobre el formato 


\begin{tabular}{lcc}
\hline \multicolumn{1}{c}{ Pregunta } & $\begin{array}{c}\text { De acuerdo o } \\
\text { totalmente de } \\
\text { acuerdo }\end{array}$ & $\begin{array}{c}\text { En desacuerdo o } \\
\text { totalmente en } \\
\text { desacuerdo }\end{array}$ \\
\hline Prefiero el formato de clase inversa al formato tradicional & $34,07 \%$ & $39,56 \%$ \\
$\begin{array}{l}\text { Creo que el formato de clase inversa debería seguir } \\
\text { usándose en los cursos siguientes en esta asignatura }\end{array}$ & $39,56 \%$ & $38,46 \%$ \\
$\begin{array}{l}\text { Creo que el formato de clase inversa debería extenderse a } \\
\text { otras asignaturas }\end{array}$ & $28,57 \%$ & $57,14 \%$ \\
$\begin{array}{l}\text { El formato de clase inversa me ha hecho sentir más } \\
\text { implicado }\end{array}$ & $35,16 \%$ & $40,66 \%$ \\
$\begin{array}{l}\text { El formato de clase inversa me ha hecho participar de } \\
\text { forma más activa }\end{array}$ & $39,56 \%$ & $37,36 \%$ \\
$\begin{array}{l}\text { El formato de clase inversa ha hecho que la asignatura me } \\
\text { resulte más interesante }\end{array}$ & $23,08 \%$ & $48,35 \%$ \\
\end{tabular}

La preferencia entre formato inverso o formato tradicional está bastante repartida, con cierta ventaja entre los que prefieren el formato tradicional. En cambio son más los que creen que la asignatura debe seguir impartiéndose en formato inverso.

En cuanto a si el formato debería extenderse o no a otras asignaturas hay una clara preferencia a favor de que no se extienda.

Con respecto a la implicación con la asignatura también son algunos más los que piensan que no se han sentido más implicados .En cambio son mayoría los que piensan que el formato les ha hecho participar de forma más activa. También opinan que el formato no ha conseguido que les interese más la asignatura.

Los alumnos creen que el formato de clase inversa ha permitido que el profesor dispusiera de más tiempo para aclarar dudas ( $42,86 \%$ frente a $27,47 \%)$.

Los alumnos consideran que el formato de clase inversa ha hecho que hayan tenido que dedicarle demasiado tiempo a la asignatura $(73,63 \%$ frente a $7,69 \%)$ con 36 totalmente de acuerdo frente a 2 totalmente en desacuerdo. Viendo los comentarios de respuesta libre este parece ser el mayor motivo de descontento con el formato.

Sólo un $27 \%$ de los 89 que han contestado dicen llevar las asignaturas al día o casi al día, casi un $35 \%$ dicen apretar el último mes y un $38 \%$ dicen que depende de la época. 
Con respecto al fomento de competencias transversales la mayoría de los alumnos no creen que el formato de clase inversa haya contribuido a fomentarlas, excepto el trabajo en equipo (ligeramente) y la capacidad de gestión de la información.

Tabla 3. Percepción del fomento de competencias

\begin{tabular}{lcc}
\hline \multicolumn{1}{c}{ Competencia } & $\begin{array}{c}\text { De acuerdo o } \\
\text { totalmente de } \\
\text { acuerdo }\end{array}$ & $\begin{array}{c}\text { En desacuerdo o } \\
\text { totalmente en } \\
\text { desacuerdo }\end{array}$ \\
\hline $\begin{array}{l}\text { Espíritu crítico y compresión de los problemas } \\
\text { relacionados con la asignatura }\end{array}$ & $26,37 \%$ & $37,36 \%$ \\
Creatividad y espíritu emprendedor & $15,38 \%$ & $48,35 \%$ \\
Capacidad de trabajo en equipo & $36,26 \%$ & $32,97 \%$ \\
Capacidad de comunicación & $32,97 \%$ & $35,16 \%$ \\
Capacidad de liderazgo & $16,48 \%$ & $41,76 \%$ \\
Cultura de la calidad & $14,29 \%$ & $38,46 \%$ \\
Capacidad de adaptación a problemas y situaciones nuevas & $29,67 \%$ & $36,26 \%$ \\
Capacidad de gestión del tiempo & $34,07 \%$ & $36,26 \%$ \\
Capacidad de gestión de la información & $40,66 \%$ & $26,37 \%$ \\
\hline
\end{tabular}

La mayoría de los alumnos dicen no haberse sentido motivados por las actividades propuestas en clase $(46,15 \%$ en desacuerdo frente a $17,58 \%$ de acuerdo) con 17 totalmente en desacuerdo frente a 1 totalmente de acuerdo.

En cambio la mayoría cree que las actividades propuestas durante las clases les han hecho reflexionar sobre el contenido del curso $(53,85 \%$ frente a $28,57 \%)$, que les han aclarado los conceptos claves de la asignatura $(61,54 \%$ frente a $16,48 \%)$, que complementan y refuerzan lo explicado en los vídeos (68,13\% frente a $15,38 \%$ ) y que sus conocimientos de Ingeniería aeroportuaria han aumentado con la asistencia a clase $(56,04 \%$ frente a $24,18 \%)$.

También creen que han permitido que la interacción con el profesor fuera más enriquecedora ( $40,66 \%$ frente a $28,57 \%$ ), que el planteamiento de las clases ha sido útil para aprender el contenido de la asignatura $(41,76 \%$ frente a $32,97 \%)$, que trabajar en grupo con sus compañeros les ha ayudado a aprender $(57,14 \%$ frente a $19,78 \%)$ y, por una ligera ventaja, que la posibilidad de exponer sus resultados a la clase les ha motivado a la hora de hacer los trabajos $(40,66 \%$ frente $36,26 \%)$.

2015, Universitat Politècnica de València 
Los alumnos creen que los vídeos les han ayudado a aprender (70,33\% frente a $17,58 \%)$, que tener la teoría en vídeo les ha facilitado estudiar la asignatura $(63,74 \%$ frente a $17,58 \%)$, que les han ayudado a asistir preparados a clase $(61,54 \%$ frente a $19,78 \%)$, que el formato de los vídeos es adecuado ( $49,45 \%$ frente a $26,37 \%)$ y que eran fáciles de entender $(79,12 \%$ frente a $6,59 \%)$.

En definitiva opinan que tener la teoría en vídeo tiene ventajas sobre el formato de clase tradicional $(71,43 \%$ frente a $13,19 \%)$.

Su opinión es que los vídeos son aburridos (53,85\% frente a18,68\%) y que deberían ser más cortos $(50,55 \%$ frente a $17,58 \%)$, algo que llama la atención si se tiene en cuenta que la duración media de los vídeos es de 3 minutos 47 segundos.

Hay un $45 \%$ que dice que tener la teoría en vídeo ha hecho que vayan menos a clase.

Se les ha preguntado a los alumnos su opinión sobre la contribución al aprendizaje de la asignatura de los materiales empleados en clase. Para comparar las respuestas se han traducido a una escala de 0 a 10: 0 nada, 2,5 poco, 5 algo, 7,5 bastante y 10 mucho:

Tabla 4. Contribución al aprendizaje

\begin{tabular}{lc}
\hline \multicolumn{1}{c}{ Pregunta } & $\begin{array}{c}\text { De acuerdo o } \\
\text { totalmente de acuerdo }\end{array}$ \\
\hline Vídeos grabados por el profesor & 7,28 \\
Transparencias colgadas en Poliformat & 6,29 \\
Entregas de problemas a realizar en casa & 6,26 \\
Feedback y aclaraciones del profesor en clase & 6,10 \\
Los problemas realizados en clase & 5,88 \\
El trabajo del plan director & 5,25
\end{tabular}

En el campo de texto libre hay comentarios a favor y en contra del formato. La queja que más se repite es que los alumnos creen que han tenido que dedicar demasiado tiempo a la asignatura y la segunda que no aprovechaban las clases porque no habían visto los vídeos antes, al no haber nada que les obligara a ello. Hay algún comentario relativo al poco tiempo disponible para ver los vídeos en algunas ocasiones y algún otro que reclama un formato algo menos "inverso" con más explicación de teoría.

\section{Resultados}

Se generado el material teórico para aplicar la metodología de clase inversa a una asignatura de 2 grupos con 120 alumnos, grabando 213 vídeos (13,5 horas), creando 25 
casos de estudio y problemas para las clases presenciales, colecciones de problemas para resolver en casa y exámenes personalizados.

Se ha generado una herramienta y un procedimiento que permite crear y subir contenido en formato tipo MOOC a PoliformaT.

Se ha creado una herramienta para crear colecciones de problemas y exámenes personalizados que se corrigen de forma automática. Esta herramienta permite generar preguntas de respuesta numérica, de texto y corrección de problemas basados en colecciones de datos personalizadas creadas con otras hojas de cálculo.

Se ha demostrado la validez y comodidad de la herramienta anterior a la hora de poner colecciones de problemas con datos personalizados que se autocorrigen.

Se ha creado y probado una dinámica de clase inversa.

Se ha realizado una encuesta de opinión a 123 alumnos que han contestado 104 de ellos (de los que 91 han opinado sobre la asignatura), con lo que se ha obtenido una valoración representativa de la percepción y opiniones de los estudiantes.

\section{Conclusiones}

Polimedia, PoliformaT y las herramientas de automatización creadas para este proyecto han permitido implementar la metodología de clase inversa en un plazo muy corto de tiempo.

La preparación del material ha supuesto un esfuerzo cuantificado en 65 horas para generar el material en vídeo y subirlo a PoliformaT y unas 85 horas en total para generar todo el material necesario para adaptar la asignatura (sin contar el desarrollo de las herramientas de automatización).

Durante las clases presenciales se genera una gran cantidad de material por parte de los estudiantes ( 25 trabajos*100 alumnos $/ 5$ alumnos por grupo $=500$ trabajos). Si se quiere incorporar su corrección a la nota de los alumnos sin que implique un gran esfuerzo por parte del profesor hay que utilizar sistemas de corrección automática (para algunos de los ejercicios se puede usar la herramienta de corrección automática creada).

La opinión del profesor es que el aprendizaje de los alumnos que se han implicado ha mejorado y que comprenden y utilizan mejor los conceptos de la asignatura.

Tras analizar los resultados de la encuesta y compararlos con las percepciones del profesor, consideramos muy importante incluir elementos de control a lo largo del curso que permitan realizar un seguimiento objetivo de la percepción de los alumnos.

La opinión de los alumnos está dividida entre los que prefieren el formato y los que no. La razón principal para rechazar el formato parece ser que los alumnos consideran que han tenido que trabajar demasiado.

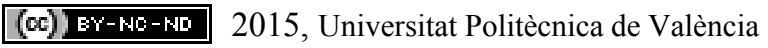


A pesar de que los alumnos dicen preferir el formato de clase tradicional, consideran que las actividades realizadas en clase han contribuido a su aprendizaje y valoran mucho el tener la teoría de la asignatura en vídeo. Las respuestas a las preguntas sobre si el formato ha contribuido a su aprendizaje indican claramente que ha sido así.

Los alumnos valoran muy positivamente disponer de vídeos con la teoría.

Aunque entre los comentarios escritos hay alguna queja sobre el poco tiempo de disponibilidad de los vídeos antes de las clases, no parece ser el inconveniente más importante. Las respuestas parecen indicar que los problemas principales son que los alumnos no están acostumbrados a dedicar el tiempo que corresponde a una asignatura y que no tienen la costumbre de autorregularse si no hay nada que les obliga a llevar la asignatura al día.

Observando las respuestas del campo de texto libre incorporaremos los siguientes cambios para el curso que viene:

En la primera clase del curso se explicará claramente cuál es la dedicación que se espera de los alumnos para una asignatura de 4,5 créditos y cómo el formato está pensado para adaptarse a esa dedicación.

Se incluirá al principio de clase un examen tipo test con preguntas muy básicas sobre los vídeos correspondientes a la semana que cuente para la nota para que los alumnos vayan preparados a las clases.

Se incluirá una parte de repaso de la teoría en cada clase presencial.

\section{Referencias}

CONEJERO, J. A., VENDRELl, E., TERRASA, A., \& SANCHIS, E. (2006). "La dedicación del alumno en la Facultad de Informática de la Universidad Politécnica de Valencia" en Actas del $4^{\circ}$ Congreso Internacional "Docencia Universitaria e Innovación"

Ministerio de Educación, Cultura y Deporte (2003). “Integración del sistema universitario español en el Espacio Europeo de Enseñanza Superior” $<\mathrm{http}: /$ tecnologiaedu.us.es $/ \mathrm{mec} 2011 / \mathrm{htm} / \mathrm{mas} / 2 / 21 / 7 . \mathrm{pdf}>$ [Consulta: 30 de mayo 2015]

TURRO, C., CAÑERO, A., y BUSQUETS, J. (2010). "Video learning objects creation with polimedia" en Multimedia (ISM), 2010 IEEE International Symposium on. IEEE. 371-376

UPV (2006). Guía docente de la UPV: Criterios para su elaboración

$<$ http://www.aqu.cat/doc/doc_52850666_1.pdf $>$ [Consulta: 30 de mayo 2015]

UPV (2007). "Plan estratégico UPV 2007-2014"

$<$ https://www.upv.es/entidades/SEPQ/infoweb/sepq/info/U0553827.pdf> [Consulta: 30 de mayo 2015]

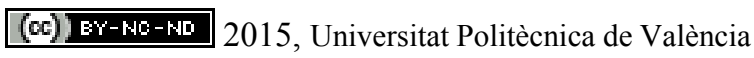

Congreso In-Red (2015) 